\title{
Charged Higgs searches in the leptonic $\tau$ channel
}

\section{Ofer Vitells ${ }^{1}$}

Weizmann Institute of Science

Rehovot, Israel

E-mail: ofer.vitells@weizmann.ac.il

\section{Eilam Gross}

Weizmann Institute of Science

Rehovot, Israel

E-mail: eilam.gross@weizmann.ac.il

Light charged Higgs bosons $\left(m_{H^{+}}<m_{t o p}\right.$ ), if exist, are expected to be produced at the LHC dominantly via top quark decays, $t \rightarrow H^{+} b$. The dominant decay mode of the charged Higgs boson in this mass range is to a $\tau$-lepton and a neutrino. We study the channel in which the $\tau$ decays leptonically (i.e. to an electron or a muon). The presence of an isolated lepton offers a cleaner experimental signature compared to hadronic $\tau$ decays, however reconstruction of the event is more challenging due to the presence of three neutrinos in the final state. We define a new transverse mass for the charged Higgs in this channel, which can be used to discriminate between the signal and the main background on a statistical basis.

Prospects for Charged Higgs Discovery at Colliders

Uppsala, Sweden

September $16^{\text {th }}-19^{\text {th }} 2008$

\footnotetext{
$1 \quad$ Speaker
} 


\section{Introduction}

Charged Higgs bosons arise in models with extended Higgs sector such as two Higgs doublets models (2HDM) and in particular the Minimal Supersymmetric Standard Model (MSSM). If the Charged Higgs mass is below the top mass, then it's dominant production mode at the LHC is expected to be via top quark decay, due to the large Yukawa coupling of the top. The dominant decay mode of the charged Higgs in this scenario is to a $\tau$ lepton $H^{-} \rightarrow \tau^{-} \bar{v}_{\tau}[1]$. It is clear therefore that the most noticeable signature of a charged Higgs would be an excess of $\tau$ leptons in top quark decays. Here we study the possibility to observe a charged Higgs using leptonic decays of the $\tau, \tau^{-} \rightarrow e^{-} \bar{\nu}_{e} \nu_{\tau}$ or $\tau^{-} \rightarrow \mu^{-} \bar{\nu}_{\mu} \nu_{\tau}$. The presence of an isolated electron or muon in the final state has an experimental advantages with regard to triggering the event and suppressing backgrounds. On the other hand, one cannot fully reconstruct the event due to the multiple-neutrino final state. We therefore investigate kinemtical properties of such events that can provide discrimination between the signal and the background on a statistical basis. In particular we define a new transverse mass for the charged Higgs in this channel. This new variable and it's properties are discussed in section 2.2.

\section{Discriminating variables}

\subsection{The $\mathrm{W}$ transverse mass}

Assuming that backgrounds other than $t \bar{t}$ can be efficiently suppressed using standard cuts, the main background to the charged Higgs signal will be the SM decay $t \rightarrow W b$ with a subsequent leptonic decay of the $\mathrm{W}$, either directly $W \rightarrow \ell v$ or via a $\tau: W \rightarrow \tau \bar{v} \rightarrow \ell \bar{v} v \bar{v}$. To distinguish between the two different background sources one might reconstruct the $\mathrm{W}$ transverse mass, defined as

$$
\left(M_{T}^{W}\right)^{2}=2 p_{T}^{\ell} p_{T}^{\text {miss }}\left(1-\cos \phi_{\ell, \text { miss }}\right)
$$

The $\mathrm{W}$ transverse mass distributions at parton level, generated using PYTHIA [2] are shown in Figure 1, for the background and a charged Higgs signal with a mass of $130 \mathrm{GeV}$. A charged Higgs signal would appear as an excess of events in the lower region of this distribution, since events with direct $\mathrm{W}$ decays contribute mainly to the Jacobian peak at the $\mathrm{W}$ mass. One should note however that as the charged Higgs mass becomes higher, the effect becomes less pronounced as the signal distribution becomes more flat. 


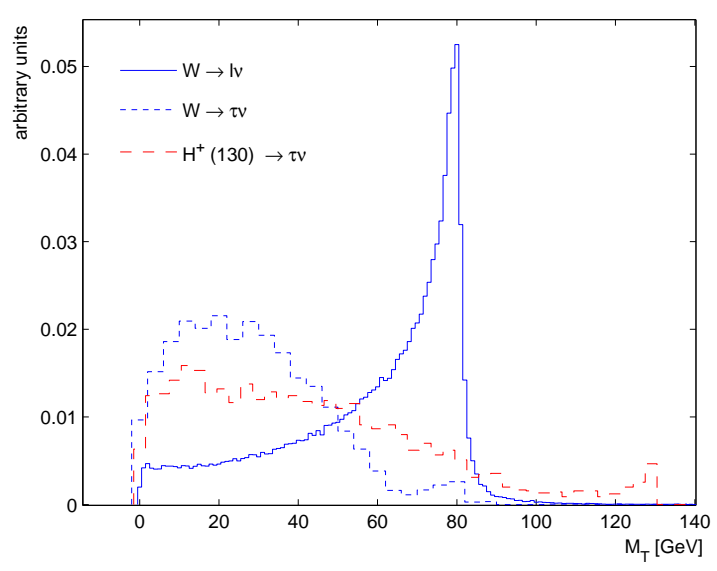

Figure 1. The parton-level $W$ transverse mass distributions for leotonic $W$ decays and a charged Higgs signal with $m_{H^{+}}=130 \mathrm{GeV}$ (all distributions are normalized to have a unit area). A minimum cut of $20 \mathrm{GeV}$ for the lepton transverse momentum is applied.

\subsection{The charged Higgs transverse mass}

The $\mathrm{W}$ transverse mass (1) can be alternatively defined in the following way:

$$
\left(M_{T}^{W}\right)^{2}=\min _{\left\{\begin{array}{c}
\text { miss } \\
p_{\text {miss }}^{\text {miss }} \\
\left(p^{\text {miss }}\right)^{2}=0
\end{array}\right\}}\left[\left(p^{\ell}+p^{m i s s}\right)^{2}\right]
$$

where $p^{\ell}, p^{\text {miss }}$ are the lepton and missing 4-momenta respectively. From this definition it follows immediately that $M_{T}^{W} \leq M_{W}$. For charged Higgs events the constraint $\left(p^{\text {miss }}\right)^{2}=0$ does not hold, however one can use a different constraint using the known value of the top mass:

$$
m_{t o p}^{2}=\left(p^{\ell}+p^{b}+p^{m i s s}\right)^{2}
$$

here $p^{b}$ is the 4-momentum of the b-jet associated to same top quark decay as does the lepton. In analogy with (2) we define the charged Higgs transverse mass as follows:

$$
\left(M_{T}^{H}\right)^{2}=\max _{\left\{\begin{array}{l}
p_{i s s}^{\text {miss }}, E^{\text {miss }} \\
\left(p^{m i s s}+p^{b}+p^{l e p}\right)^{2}=m_{\text {top }}^{2}
\end{array}\right\}}\left[\left(p^{\ell}+p^{\text {miss }}\right)^{2}\right]
$$

Note that in contrast with (2), here the transverse mass is defined as a maximum rather then a minimum, which is due to the fact that the constraint (3) impose an upper bound for the expression $\left(p^{\ell}+p^{m i s s}\right)^{2}$.It follows that the charged Higgs transverse mass satisfies:

$$
m_{H^{+}} \leq M_{T}^{H} \leq m_{\text {top }}
$$


The explicit expression for $M_{T}^{H}$ is given by (see appendix A):

$$
\left(M_{T}^{H}\right)^{2}=\left(\sqrt{m_{t o p}^{2}+\left(\vec{p}_{T}^{\ell}+\vec{p}_{T}^{b}+\vec{p}_{T}^{\text {miss }}\right)^{2}}-p_{T}^{b}\right)^{2}-\left(\vec{p}_{T}^{\ell}+\vec{p}_{T}^{\text {miss }}\right)^{2}
$$

In the derivation of $M_{T}^{H}$ we used the constraint (3), namely that the invariant mass of the sum of the lepton, b-jet and missing momenta equals the top mass. In reality, this relation is not exact since top quark decay can involve gluon radiation [3]. In that case we have:

$$
\sqrt{\left(p^{\ell}+p^{b}+p^{m i s s}\right)^{2}}=m_{t o p}^{*} \leq m_{t o p}
$$

therefore, since $M_{T}^{H}$ is a monotonically increasing function of $m_{\text {top }}$ :

$$
M_{T}^{H}\left(m_{t o p}\right) \geq M_{T}^{H}\left(m_{t o p}^{*}\right) \geq m_{H^{+}}
$$

i.e. relation (1.5) still holds, however the values of $M_{T}^{H}$ will be shifted upwards and thus the Jacobian peak will be smeared. This effect is shown in Figure 2, where the distribution of $M_{T}^{H}$ is shown for the normal case (2a) and a hypothetical one in which there is no radiation $(2 b)$.

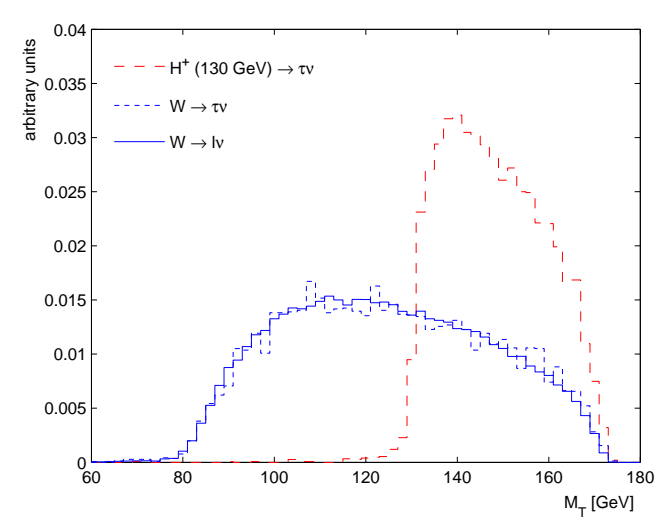

(a)

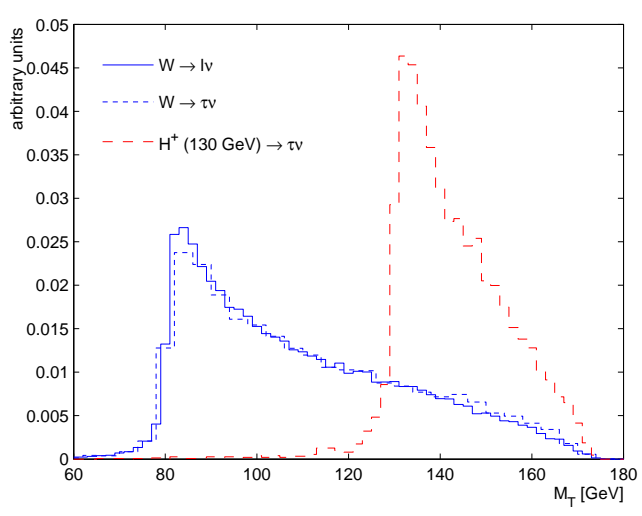

(b)

Figure 2. Distributions of the charged Higgs transverse mass with (a) and without (b) the effect of gluon radion from the top quark decay. The distributions are shown for a charged Higgs with a mass of 130 $\mathrm{GeV}$ (dashed red) and leptonic $W$ decays (solid and dotted blue).

A feature of the $M_{T}^{H}$ distribution that can be seen in Figure 2 is that it has the same shape for both $\mathrm{W}$ decay modes. This is easily understood from expression (1.6), where the dependance on the lepton and missing transverse momenta is only through their sum $\vec{p}_{T}^{\ell}+\vec{p}_{T}^{\text {miss }}=\vec{p}_{T}^{W}$. Namely $M_{T}^{H}$ depends on the $\mathrm{W}$ transverse momentum which has the same distribution regardless of the $\mathrm{W}$ decay mode.

Clearly, the discrimination between the signal and the $\mathrm{W}$ background becomes stronger as the charged Higgs mass is higher, in contrast to the $\mathrm{W}$ transverse mass discussed in the previous section. Thus the two variables are complementary in providing sensitivity to a charged Higgs. 


\section{Conclusions}

The leptonic decay channel of the $\tau$ could be used to increase the sensitivity to a charged Higgs in $t \bar{t}$ events. This might be particularly important at early stages of LHC data, before hadronic $\tau$ reconstruction is well understood. We have demonstrated that by using appropriate kinematical observables it is possible to distinguish between a charged Higgs and a W boson on a statistical basis. In particular we have defined a new transverse mass for the charged Higgs in this channel, which provides powerful discrimination between the signal and the background.

\section{Appendix}

\section{A. Derivation of $M_{T}^{H}$}

In this appendix, we derive the expression for the charged Higgs transverse mass. Our goal is to maximize the expression of invariant mass:

$$
m_{H^{+}}^{2}=\left(p^{\ell}+p^{m i s s}\right)^{2}
$$

with respect to the two unknown components of $p^{\text {miss }}$, while holding the constraint:

$$
m_{\text {top }}^{2}=\left(p^{m i s s}+p^{\ell}+p^{b}\right)^{2}
$$

For convenience, we adopt the notation $p_{\|}=\left(E, p_{z}\right)$, satisfying $p_{\|}^{2}=E^{2}-p_{z}^{2}$, and $\vec{p}_{T}=\left(p_{x}, p_{y}\right)$ with $\vec{p}_{T}^{2}=p_{x}^{2}+p_{y}^{2}$. Equation (A.1) and (A.2) can be re-written as:

$$
\begin{gathered}
m_{H^{+}}^{2}=\left(p_{\|}^{\ell}+p_{\|}^{\text {miss }}\right)^{2}-\left(\vec{p}_{T}^{\ell}+\vec{p}_{T}^{\text {miss }}\right)^{2} \\
m_{\text {top }}^{2}=\left(p_{\|}^{\ell}+p_{\|}^{\text {miss }}+p_{\|}^{b}\right)^{2}-\left(\vec{p}_{T}^{\ell}+\vec{p}_{T}^{\text {miss }}+\vec{p}_{T}^{b}\right)^{2}
\end{gathered}
$$

To maximize Eq. (A.3), we introduce a Lagrange multiplier $\lambda$ and differentiate with respect to $p_{\|}^{\text {miss }}$ :

$$
\frac{\partial}{\partial p_{\|}^{\text {miss }}}\left(\left(p_{\|}^{\ell}+p_{\|}^{\text {miss }}\right)^{2}-\left(\vec{p}_{T}^{\ell}+\vec{p}_{T}^{\text {miss }}\right)^{2}\right)=0
$$

This gives

$$
p_{\|}^{\text {miss }}=\frac{\lambda}{1-\lambda} p_{\|}^{\ell}-p_{\|}^{b}
$$

plugging this into Eq. (A.4) we obtain for $\lambda$ :

$$
1-\lambda=\frac{p_{T}^{b}}{\sqrt{m_{\text {top }}^{2}+\left(\vec{p}_{T}^{\ell}+\vec{p}_{T}^{\text {miss }}+\vec{p}_{T}^{b}\right)^{2}}}
$$

where we have approximated the b-quark to be massless. Plugging (A.7) and (A.6) into (A.3) we obtain the final result:

$$
\left(M_{T}^{H}\right)^{2}=\left(\sqrt{m_{\text {top }}^{2}+\left(\vec{p}_{T}^{\ell}+\vec{p}_{T}^{b}+\vec{p}_{T}^{\text {miss }}\right)^{2}}-p_{T}^{b}\right)^{2}-\left(\vec{p}_{T}^{\ell}+\vec{p}_{T}^{\text {miss }}\right)^{2}
$$


It should be noted that exactly the same procedure could be applied to the case of a leptonic $\mathrm{W}$ decay $W \rightarrow \ell v$, by replacing the constraint (A.2) with $\left(p^{\text {miss }}\right)^{2}=0$. This will result in the usual expression for the $\mathrm{W}$ transverse mass.

Finally, it's worth noting that from this point of view the existence of a Jacobian peak at the edge of the distribution follows immediately. For example, we have:

$$
\frac{d \sigma}{d M_{T}^{H}}=\frac{d \sigma}{d E_{\text {miss }}}\left(\frac{d M_{T}^{H}}{d E_{\text {miss }}}\right)^{-1}
$$

and by construction, $\left.\frac{d M_{T}^{H}}{d E_{m i s s}}\right|_{M_{T}^{H}=m_{H^{+}}}=0$.

\section{References}

[1] M. Carena, J. S. Conway, H. E. Haber, J. D. Hobbs, et al, Report of the Higgs working group of the Tevatron run 2 SUSY/Higgs workshop, [hep-ph/0010338].

[2] T. Sjöstrand, S. Mrenna, P. Skands, JHEP 05 (2006) 026.

[3] L.H. Orr, T. Stelzer, W.J. Stirling, Gluon radiation and Top quark physics, Phys. Rev. D 56 (1997) 1, [hep-ph/9609354v1]. 